\title{
Photosynthetic Constraints on the Habitable Zone
}

\author{
Michael C. Storrie-Lombardi \\ Jet Propulsion Laboratory, California Institute of Technology, 4800 Oak \\ Grove Drive, $M / S$ 183-301, Pasadena, California 91109, U.S.A.
}

Kinohi Institute, 530 South Lake Avenue, Pasadena, California 91101, U.S.A.

\author{
A. I. Tsapin, G. D. McDonald, M. L. Coleman \& V. S. Meadows \\ Jet Propulsion Laboratory, California Institute of Technology, 4800 Oak \\ Grove Drive, Pasadena, California 91109, U.S.A.
}

\begin{abstract}
The search for extraterrestrial habitable planets will require long observation times and the intelligent selection of appropriate parent stars and target biosignatures. While life can certainly develop in the absence of photosynthesis, such life forms on earth exhibit metabolic rates several orders of magnitude less than the activity accompanying a photosynthetic-driven ecosystem. The most accessible spectral biosignatures are those accompanying a system driven away from thermodynamic equilibrium by photosynthetic activity. For example, the co-existence in a planetary atmosphere of significant amounts of ozone, oxygen, and methane would be a strong indication of biotic activity. Investigating the issue of the Habitable Zone from the standpoint of the constraints inherent in photosynthesis it appears that the absorption characteristics of photosynthetic microorganisms on this planet make it likely that photosynthetic activity can exist on planets orbiting stars to red-ward of the Sun on the H-R diagram. Such a possibility is encouraging for terrestrial planet finder efforts since stars classified red-ward of our sun (G3 to K7) account for more than $55 \%$ of our nearest neighbors.
\end{abstract}

\section{Introduction}

The habitable zone (HZ) around a star has been previously defined as the region in which an Earth-like planet could maintain liquid water (Kasting et al. 1993). Earth, our only known example of an inhabited planet, orbits a middle-aged G2 dwarf star with an estimated lifespan of $10 \mathrm{~Gy}$. Evidence from the molecular biology community supports the early appearance and evolution of photosynthesis on Earth (Xiong et al. 2000). While G stellar types are numerous, they are not the "average" classification of the H-R diagram main sequence stars. Figure 1 depicts the distribution of main sequence types found within $25 \mathrm{pc}$ of Earth (data adapted from Ames NASA/NSF Near Star catalog). The majority of objects appear as cooler stars than Sol, with temperatures ranging between $5800 \mathrm{~K}$ and $5300 \mathrm{~K}$ for G3 to G9 stars and between 5300 and $4000 \mathrm{~K}$ for $\mathrm{K} 0$ 
to K7 stars. Stars classified red-ward of our sun as G3 to K7 account for more than $55 \%$ of observed objects. Within the next decade the active search for terrestrial planets orbiting within $\mathrm{HZ}$ will require observation of relatively near targets. If we confine our search to "Sol-like" stars, e.g. G1 and G2 stellar types, we have a total of 32 targets within 25 pc of Earth. In contrast, if the existence of $\mathrm{HZ}$ appears possible for G3-K7 stars, inclusion of these objects would add 362 nearby targets for Terrestrial Planet Finder (TPF).

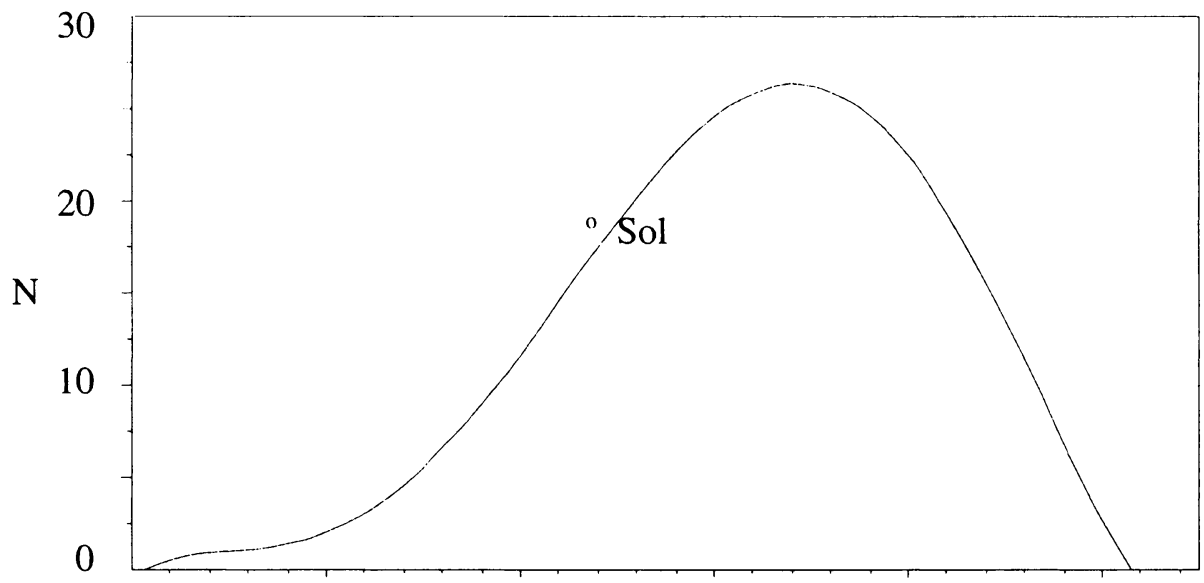
A $13357 \mathrm{~F} 13557 \mathrm{G} 13557 \mathrm{~K} 11357 \mathrm{M} 133579$
Classification

Figure 1. Number of stars $(\mathrm{N})$ as a function of stellar types in 663 main sequence stars less than 25 parsecs from Sol (data from the Ames NASA/NSF Near Star catalog, http://nstars.arc.nasa.gov). More than $55 \%$ of stars are classified as types G3 to K7.

\section{Habitable Zone Parameters}

Unfortunately, a number of unresolved issues accompany the shift in focus to redder main sequence stars (Kasting et al. 1993). Spectral reddening, diminished mass, and increased longevity of the star accompany the decrease in stellar surface temperature and luminosity. The primary relationships between mass, longevity, luminosity, and the orbital change required to compensate for diminished luminosity are depicted in Fig. 2 below.

Moving from stellar type G2 to $\mathrm{K} 5$ is accompanied by a $1 / 3$ diminution mass and a decrease in luminosity to $\sim 10 \%$ that available to Earth. To obtain Earth-equivalent flux a decrease in orbit to $0.4 \mathrm{AU}$ is required. The impact of these environmental changes on the probability of terrestrial planet formation and the subsequent origin and evolution of life on a terrestrial planet in these systems is unknown. To fine tune the constraints on the habitable zone for terrestrial planet finder efforts will require investigation into the effect of diminished 


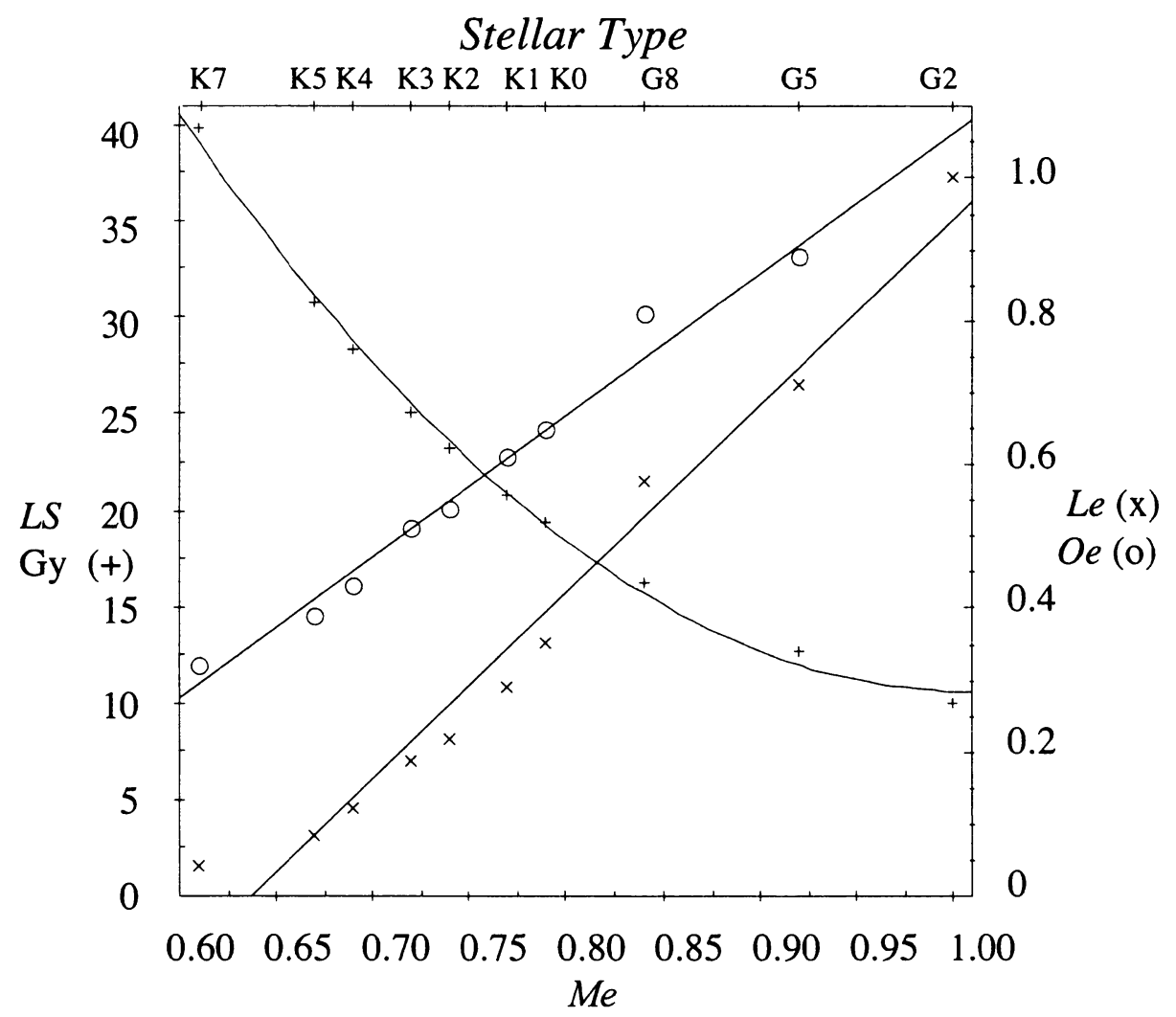

Figure 2. Stellar spectra from main sequence stars $<25 \mathrm{pc}$ from Earth exhibit a decrease in luminosity as a function of diminished mass. At an Earth equivalent mass $(\mathrm{Me})$ of 0.64 a $\mathrm{K} 5$ star has an Earth equivalent luminosity $(L e)$ of 0.10 . Compensation for the loss in solar flux requires an Earth equivalent orbit for a terrestrial planet of $0.4 \mathrm{AU}$. Estimated life span $(L S)$ of the K5 star would be $\sim 30 \mathrm{~Gy}$.

planetary mass and ultraviolet flux on atmosphere evolution and retention; the probable evolution of vulcanism and geochemical weathering in low mass environments; and the impact of decreased material for planetary formation in low mass stellar systems. In this work we address only one of the myriad of effects: the impact of spectral reddening on photosynthesis. Moving down the main sequence of stars from $F$, through $G$, to $K$ stellar types produces the spectral shifts depicted in Fig. 3. The overall effect is an increase flux in the red end of the spectra and a decrease in the blue, i.e. a "reddening" of the spectra. It is this shift in available photon energy that is central to our understanding of the photosynthetic constraints on the habitable zone, not the total flux available. Shifting the required orbital radius for the habitable zone towards the parent low mass star provides compensation for total flux but does not effect the changes induced by the spectral shift. 


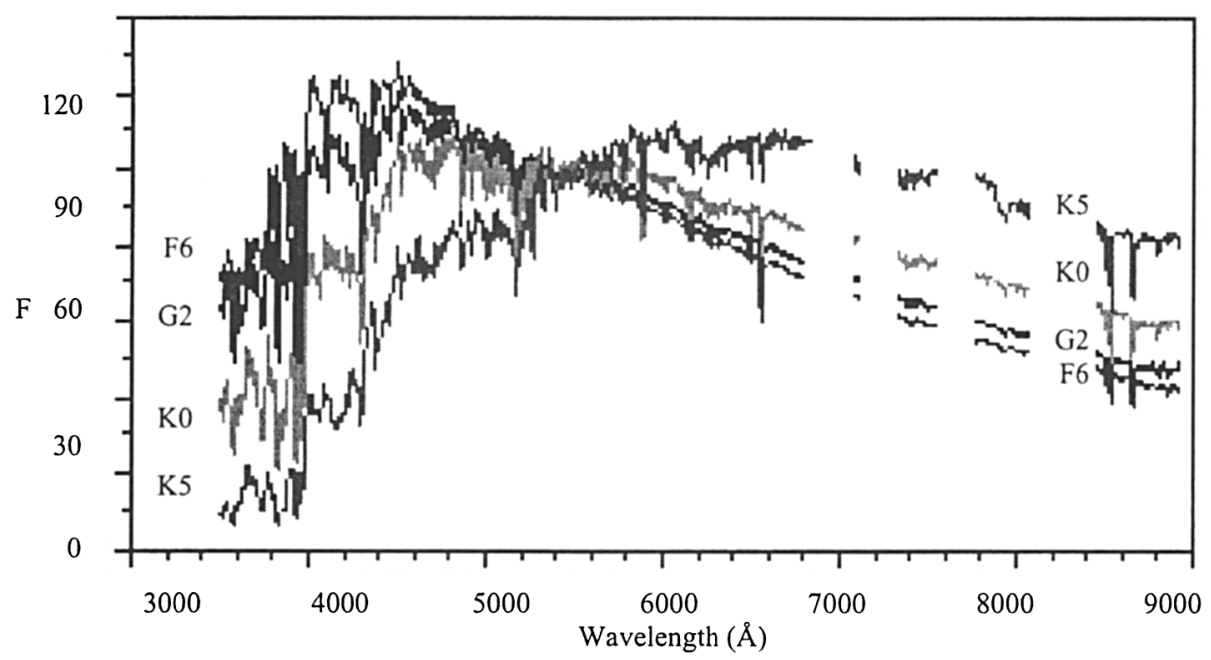

Figure 3. Stellar spectra from main sequence stars exhibit a redward shift in the shape of the continuum across F7 to K5 stellar types. The shift is due to decreasing stellar surface temperature, a parameter con-trolled primarily by stellar mass.

\subsection{Photosynthesis}

Photosynthesis in an organism begins with the efficient capture of solar photons. To accomplish this, plants, bacteria, and algae have produced a variety of pigments optimized for the absorption of particular wavelengths of light. Often, a single organism will contain multiple pigments and proteins working together in what is called a light harvesting complex (LHC). LHCs in terrestrial organisms include photon-absorbing pigments optimized for a wide variety of light conditions. Shifts in the spectral distribution of the solar flux received on Earth can be expected to play a critical role in the evolution of the constituent molecules of these complexes. The faint young sun hypothesis suggests that our own sun was significantly cooler and redder early in the life of our planet. This raises the possibility that understanding the light harvesting characteristics of the early inhabitants of Earth might be of assistance in predicting photosynthetic constraints on the HZ of low mass stars. Current estimates place the earliest appearance of microbial life at $\sim 3.3 \mathrm{~Gy}$ (Schopf, 1999). This is early enough for microorganisms to experience growth and evolution under a cooler, redder sun. Two of the most ancient lineages, the purple bacteria and cyanobacteria, appear to have LHCs capable of performing in such an environment. While the stellar spectral shift seen in low mass stars would be disadvantageous to organisms employing pigments with absorption maxima in the blue, LHCs that include pigments operating efficiently in the red and near-IR would be relatively unaffected. Chlorophyll a and b exhibit dual absorption maxima at 4300-6620 and $4530-6420$, respectively. While the blue pigments would be of diminished utility in a low mass star environment, the red-absorbing pigments would remain effective. Cyanobacteria contain phycobiliproteins with absorption maxima ranging 


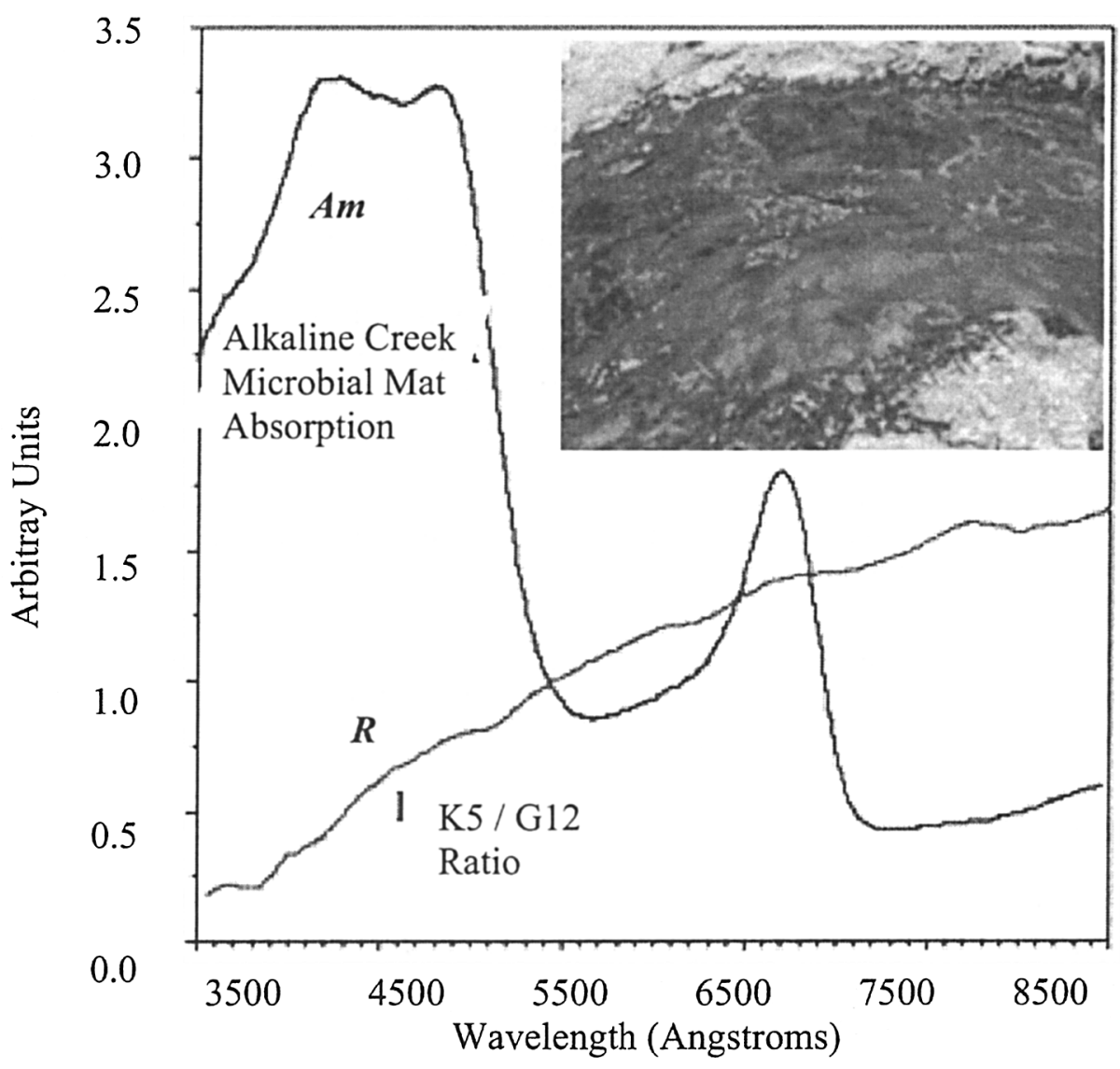

Figure 4. The spectral shift represented by the normalized ratio (R) of a typical K5 spectrum to a G2 spectrum I superimposed on the absorption spectrum $(\mathrm{Am})$ of a microbial mat community living in an alkaline mountain stream. This community of organisms exhibits a strong absorption pattern in the blue, but also reveals the typical strong secondary peak in the red. The blue absorption would be most advantageous for organisms living on planets bathed in the light of a hotter, bluer star. The secondary red absorption ability would be particularly advantageous for life on stars red-ward of the Sun.

between 5450 (B-phycoerythrin) and 6150 (C-phycocyanin). Purple bacteria contain LHCs comprised of multiple pigments and proteins. Carotenoids frequently are included in these complexes to provide absorption in the blue and green regions. Other pigments have absorption maxima shifted far into the red such as the LHCs absorbing at 8000-8500 um found in R. acidophilus seen in Fig. 4. A novel LH2 complex has been isolated from Rps. palustris that absorbs at $8000 \mathrm{um}$ (Tharia et al. 1999). Under low light growth conditions these molecule become the dominant LH2 complex for the organism. The relative shift 
in spectral power density that occurs as we move red-ward along the main sequence is depicted in Fig. 4 and compared to the absorption spectra of a mixed microbial population found in highly alkaline mountain stream. The relative spectral shift is demonstrated by a simple ratio of the K5 and G2 spectra presented in Fig. 4. The shift is characterized by a marked decrease in UV and blue flux, and a relative increase in red and infrared power. Certainly for organisms such as these, the shift to a faint red sun would have relatively minimal impact on light harvesting ability. As a result of these preliminary observations we have initiated an investigation into molecular phylogenetic and light harvesting characteristics a diverse set of photosynthetic microorganisms. The long-range goal of the effort is to constrain both habitable zone estimates of nearby stellar systems to aid terrestrial planet finder efforts and the faint young sun hypothesis for our own solar system. It is important to state again that this is only one of many parameters required to adequately constrain $\mathrm{HZ}$ estimates prior to initiating TPF observational work. Observing time requirements for each observation will be significant and proper prioritization of nearby targets critical for successful conduct of this search.

Acknowledgments. The senior author would like to thank the NASA Astrobiology Institute, the Kinohi Institute, and the Master and Fellows of Clare College, Cambridge University for their ongoing support and encouragement.

\section{References}

Cogdell, R., \& Isaacs, N. W. 1996, Structure, 4, 449

Kasting, J. F., Whitmire, D. P., \& Reynolds, R. T. 1993, Icarus, 101, 108

Schopf, J. W. 1999, Cradle of Life: The Discovery of earth's Earliest Fossils, (Princeton U. Press), 367

Tharia, A. et al. 1999, Photosyn. Res., 61, 157

Xiong, J., Fischer, W. M., Inoue, K., Nakahara, M., \& Bauer, C. E. 2000, Science, 289, 1724 